\title{
Electrical Stimulation of Spared Corticospinal Axons Augments Connections with Ipsilateral Spinal Motor Circuits after Injury
}

\author{
Marcel Brus-Ramer, ${ }^{1}$ Jason B. Carmel, ${ }^{1,2}$ Samit Chakrabarty, ${ }^{1}$ and John H. Martin ${ }^{1,3,4}$ \\ Departments of ${ }^{1}$ Neuroscience, ${ }^{2}$ Neurology, ${ }^{3}$ Neurological Surgery, and ${ }^{4}$ Psychiatry, Columbia University and New York State Psychiatric Institute, New \\ York, New York 10032
}

\begin{abstract}
Activity-dependent competition shapes corticospinal (CS) axon outgrowth in the spinal cord during development. An important question in neural repair is whether activity can be used to promote outgrowth of CS axons in maturity. After injury, spared CS axons sprout and make new connections, but often not enough to restore function. We propose that electrically stimulating spared axons after injury will enhance sprouting and strengthen connections with spinal motor circuits. To study the effects of activity, we electrically stimulated CS tract axons in the medullary pyramid. To study the effects of injury, one pyramid was lesioned. We studied sparse ipsilateral CS projections of the intact pyramid as a model of the sparse connections preserved after CNS injury. We determined the capacity of CS axons to activate ipsilateral spinal motor circuits and traced their spinal projections. To understand the separate and combined contributions of injury and activity, we examined animals receiving stimulation only, injury only, and injury plus stimulation. Both stimulation and injury alone strengthened CS connectivity and increased outgrowth into the ipsilateral gray matter. Stimulation of spared axons after injury promoted outgrowth that reflected the sum of effects attributable to activity and injury alone. CS terminations were densest within the ventral motor territories of the cord, and connections in these animals were significantly stronger than after injury alone, indicating that activity augments injury-induced plasticity. We demonstrate that activity promotes plasticity in the mature CS system and that the interplay between activity and injury preferentially promotes connections with ventral spinal motor circuits.
\end{abstract}

Key words: corticospinal; spinal motor circuits; motor cortex; plasticity; spinal cord injury; sprouting

\section{Introduction}

During development, corticospinal (CS) axon outgrowth in the spinal cord is shaped by activity-dependent competition. More active CS axons from one hemisphere overgrow regions of the spinal cord normally occupied by less active CS axons from the other hemisphere (Friel and Martin, 2005; Martin, 2005). Limiting CS activity from one hemisphere during development results in functional deficits of the contralateral forelimb (Friel et al., 2007) that can be ameliorated by limiting activity from the other hemisphere later in development (Friel and Martin, 2007). Thus, activity can be manipulated during development to stunt or promote CS function.

Here we ask whether activity also can promote outgrowth of CS axons after partial injury in maturity. After injury, spared CS axons sprout in the brainstem and spinal cord (Aoki et al., 1986; Hill et al., 2001; Weidner et al., 2001) and make new connections

Received Aug. 1, 2007; revised Aug. 30, 2007; accepted Sept. 25, 2007.

This work was supported by the Christopher and Dana Reeve Foundation, the New York State Spinal Cord Injury Research Board, and National Institutes of Health Grants 5 K12 NS001698-09 and GM 07367. We thank Xiu Li Wu for tracer histochemistry, Dan Raz for developing the imaging software in Matlab, Dr. M. Osman for veterinary care, and Erin Nunnik and Cedrick Mendoza-Tolentino for technical assistance.

Correspondence should be addressed to Dr. John H. Martin, Department of Neuroscience, Columbia University, 1051 Riverside Drive, New York, NY 10032. E-mail: jm17@columbia.edu.

DOI:10.1523/JNEUROSCI.3489-07.2007

Copyright $\odot 2007$ Society for Neuroscience 0270-6474/07/2713793-09\$15.00/0
(Bareyre et al., 2004). Novel circuits after CS injury drive spontaneous functional improvement after CS injury (Weidner et al., 2001). Harnessing activity-dependent plasticity might be a way to restore lost connections between the brain and spinal cord after injury.

In this study, we addressed two questions about activity and injury in the mature CS system. First, what are the contributions of activity and injury driving CS system plasticity? Second, does activity-dependent plasticity cooperate with injury-induced sprouting of spared CS axons to strengthen their connections with spinal motor circuits? To study the effects of activity, we electrically stimulated CS tract axons in the medullary pyramid, which contains all of the tract's axons from one hemisphere. To study injury, one pyramid was lesioned. The pyramid contains predominantly contralateral (90\%) and sparse ipsilateral CS axons (10\%) (Joosten, 1997; Brosamle and Schwab, 2000). We focused on the ipsilateral projection of the intact pyramid, whose connections and physiological actions were measured. This models the sparse CS connections preserved after CNS injury, such as stroke or spinal injury.

To understand the separate and combined contributions of injury and activity, we examined animals receiving stimulation only, injury only, and injury plus stimulation. We determined the capacity of CS axons to activate ipsilateral spinal motor circuits and traced their projections to the cord. Our hypotheses stem 
from the developmental competition model (Martin and Lee, 1999; Salimi et al., 2004; Martin, 2005). We predicted that both injury alone, because of loss of competition from the lesioned contralateral projection, and stimulation alone, by outcompeting less active terminations, would cause outgrowth of ipsilateral CS axons and stronger ipsilateral motor responses. We further predicted that the effects of injury and activity would cooperate.

We found that both stimulation and injury alone strengthened ipsilateral CS connectivity. The greatest effect, however, was seen with stimulation after injury. CS terminations in these animals were densest within the ventral motor territories of the cord, and these connections were significantly stronger than after injury alone. This indicates that activity can augment sparse connections from motor cortex to ipsilateral spinal motor circuits. Using activity to drive plasticity of spared CS axons could help restore motor function after brain or spinal injury.

\section{Materials and Methods}

Experiments were conducted on adult female Sprague Dawley rats. The surgeries (tracing, electrode implantation, and spinal electrophysiology) were performed under general anesthesia $(80 \mathrm{mg} / \mathrm{kg}$ ketamine; $10 \mathrm{mg} / \mathrm{kg}$ xylazine, i.p.) and sterile conditions. All procedures were approved by the institutional animal use committee.

\section{Corticospinal axon tracing}

Two weeks before pyramidal tract (PT) lesion (PTx) and/or electrode implantation, corticospinal tract (CST) axons were labeled bilaterally by pressure injecting (Picopump, World Precision Instruments, Sarasota, $\mathrm{FL}$ ) the tracer biotinylated dextran amine (BDA; Invitrogen, Carlsbad, CA; $10 \%$ in $0.1 \mathrm{M} \mathrm{PBS} ; 300 \mathrm{~nL} /$ injection site) into one motor cortex and Lucifer yellow-dextran amine (LY-DA; Invitrogen; 1\% in PBS; $300 \mathrm{~nL} /$ injection site) in the other. We made 15 BDA injections, separated by $\sim 500 \mu \mathrm{m}$, into the stimulated and/or intact hemisphere and 3 LY-DA injections into the lesioned and/or unstimulated side. Injections were made $1.5 \mathrm{~mm}$ below the pial surface. This covered the forelimb area of sensorimotor cortex and rostral motor field on each side (Neafsey et al., 1986). Using separate tracers for each hemisphere allowed identification of stimulated and nonstimulated axon terminations. For severed axons in the lesioned PT, LY-DA tracing provided evidence that the lesion severed all of the CST axons from one hemisphere; histology showed no LY-DA labeling in the caudal brainstem or spinal cord of lesioned animals. BDA was used to trace the CS axons that were measured in the ipsilateral cervical spinal cord. Animals recovered for 2 weeks before PTx or implantation of stimulating electrodes, ensuring that there were no impairments caused by the tracer injections.

\section{Pyramidal tract lesion and electrode implantation}

Four animal groups were used: uninjured controls, PTx alone, stimulation alone, and PTx followed by stimulation. A small craniotomy was made in the ventral surface of the occipital bone exposing the PT on the ventral brainstem surface. For PTx, a $1.1 \mathrm{~mm}$ wire knife (diameter of knife arc; Kopf Instruments, Tujunga, CA) was lowered to a depth of 1.1 $\mathrm{mm}$ below the ventral medulla surface [ $4 \mathrm{~mm}$ caudal to interaural distance (Paxinos and Watson, 1986)] immediately adjacent to the basilar artery. The wire was deployed and gently lifted to the surface, ensuring a complete lesion of one pyramidal tract. Lesions were verified histologically by transverse sections at the lesion site (supplemental Fig. S1, available at www.jneurosci.org as supplemental material).

For PT stimulation, a tripolar electrode (two leads, one ground; 0.005 inch insulated stainless steel; PlasticsOne, Roanoke, VA) was placed on the surface of the PT. Correct placement was verified at the time of implantation by evoking selective movement of the contralateral forelimb with a threshold electrical stimulus (typically 35-120 $\mu \mathrm{A}$ ). The electrode was then secured with dental acrylic adhered to two microscrews (Small Parts, Miami Lakes, FL) anchored to bone. Immunostaining for GFAP showed no evidence of gliosis or other tissue injury within the pyramid. Although the presence of the electrode sometimes caused a distortion in the pyramid surface, we were further able to confirm that there was no damage, because we followed labeled axons through the implantation site and saw no loss of labeling. The electrode was attached to a connector mounted to the dorsal skull surface with acrylic cement and bone screws. Animals in the injured plus stimulated group underwent both procedures during one surgery. Animals in the control group underwent a surgery that included craniotomy and electrode implantation but without stimulation ("sham stimulation").

Our approach, using an electrode mounted to the ventral pyramid surface, selectively activated the CST based on two control experiments (for further discussion, see supplemental material 3, available at www. jneurosci.org). First, lesion of the stimulated pyramid in acute control experiments abolished the selective contralateral motor response evoked by PT stimulation (supplemental Fig. S3A,B, available at www. jneurosci.org as supplemental material). Second, surface stimulation at higher currents after PT lesion in acute control experiments, or from within the reticular formation dorsal to the pyramid using a macroelectrode (Microprobe, Gaithersburg, MD), evoked responses that were substantially shorter than the responses evoked in the four animal groups (supplemental Fig. S3C, available at www.jneurosci.org as supplemental material). The shorter responses are consistent with activation of reticulospinal axons (for further description, see supplemental material 3, available at www.jneurosci.org).

\section{Stimulation parameters}

Animals receiving stimulation were attached to a commutator (PlasticsOne) and stimulated $6 \mathrm{~h}$ each day for $10 \mathrm{~d}$. We used a constant-current stimulator (A-M Systems, Sequim, WA) with the following stimulation parameters: $333 \mathrm{~Hz}, 45 \mathrm{~ms}$ burst, delivered every $2 \mathrm{~s}$. We chose these parameters because we found them to be optimal for evoking spinal postsynaptic responses (Meng et al., 2004) and limb movement. Stimulation current was set at the minimum level needed to evoke forelimb motor contraction. At the end of the stimulation period, animals were given an overdose of anesthetic and perfused (see below). Stimulation points in the pyramid are verified using the Prussian blue reaction for iron deposits. Rats moved about their cages less during the $6 \mathrm{~h}$ stimulation period. Because the current was set at the threshold for the smallest visible movement, the animals appeared unperturbed by the stimulation. Moreover, there were no signs of limb disuse or behavioral deficit observed after stimulation.

\section{Motor nerve recordings}

Rats underwent a terminal electrophysiological evaluation of the electroneurogram (ENG) recorded from the deep radial nerve (DRN). Animals were anesthetized using ketamine, which preserves corticospinal evoked motor responses (Hicks et al., 1992; Sloan, 1998). Nerve cuff recording electrodes (as described by Julien and Rossignol, 1982), made of molded Reprosil (Dentsply, Milford, DE) and braided stainless steel wire (CFW, Grover Beach, CA), were placed on the left and right radial nerves just distal to the bifurcation into the deep and superficial branches. Cuffs were secured to the nearby triceps tendon and covered with Reprosil to insulate the nerve and recording wires. The implanted ventral PT electrode (see above) was used to evoke responses in contralateral and ipsilateral DRNs. We recorded responses from the cuff electrodes using a differential amplifier (model 1800; AM Systems, Carlsborg, WA) (gain $1000 \times$, high-pass filter, $10^{4} \mathrm{~Hz}$, low-pass filter, $10 \mathrm{~Hz}$ ). We used different numbers of pulses to control for effects of summation. For each stimulus train (two, three, five, or eight pulses, $333 \mathrm{~Hz}$ ), we determined the minimum current needed to evoke a contralateral and an ipsilateral ENG response (e.g., "threshold") in at least $50 \%$ of the trials. We acquired the responses using an analog-to-digital converter (Digidata 1322A; Axon Instruments, Sunnyvale, CA) at $10-20 \mathrm{kHz}$ per channel. After each experiment, we verified the threshold response off-line using the program Axograph for OS X (Axograph Scientific, Sydney, Australia) for the Macintosh computer (Apple Computer, Cupertino, CA). Supplemental Figure S2 (available at www.jneurosci.org as supplemental material) shows a representative sample recording from the DRN from a stimulation-only animal evoked by PT electrode stimulation.

Threshold currents for contralateral and ipsilateral ENG responses were obtained in response to each of the four stimulus trains. To compare 
between animals, we computed a ratio of the ipsilateral to the contralateral threshold. Using a ratio eliminates animal-dependent state differences, such as depth of anesthesia and stimulating electrode characteristics. Average threshold ratios for each stimulus train were computed for each animal group. This ratio was used as a measure of the relative ability to activate ipsilateral spinal motor circuits.

Repeated measures ANOVA and the Games-Howell post hoc test (5\% significance level) were used to determine differences among groups using the program Statview (SAS, Cary, NC) for the Macintosh computer. Traces of ENG activity were also analyzed for onset latency (time from first stimulus artifact to ENG response onset) in response to a three-pulse stimulus train. Latencies were measured from the average of traces. Images of traces were constructed from averages of responses.

\section{Tissue preparation and staining}

At the end of the survival period, animals were killed and perfused with saline followed by $4 \%$ paraformaldehyde. The brain and spinal cord were removed, postfixed in the same fixative at room temperature for $1.5-2 \mathrm{~h}$, and then transferred to $20 \%$ sucrose in $0.1 \mathrm{M}$ phosphate buffer at $4^{\circ} \mathrm{C}$ overnight. Frozen transverse sections $(40 \mu \mathrm{m})$ from the sixth cervical segment were cut serially and collected in a $0.1 \mathrm{~m}$ PBS solution. We also collected sections through the pyramid, to verify electrode placement and/or lesion, and the motor cortex, to verify tracer injection locations.

Sections were processed for BDA histochemistry and LY immunohistochemistry to determine the distribution of labeled CS terminals. Selected spinal, medullary, and cortical sections were Nissl stained to assess cortical/laminar cytoarchitecture and the lesion site. For visualization of $\mathrm{BDA}$, sections were incubated in $\mathrm{PBS}, \mathrm{pH} 7.4$, containing $1 \%$ avidinbiotin complex reagent (ABC kit; Vector Laboratories, Burlingame, $\mathrm{CA}$ ) and $0.2 \%$ Triton $\mathrm{X}-100$ for $2 \mathrm{~h}$ at room temperature. After rinsing, sections were incubated with the chromogen diaminobenzidine (DAB; Sigma, St. Louis, MO) for 6-30 min. After rinsing again, sections were mounted on gelatin-coated slides, air-dried overnight, dehydrated, and coverslipped. For visualization of LY, sections were incubated at $4^{\circ}$ overnight in PBS, pH 7.4, containing $0.01 \%$ rabbit anti-LY antibody (Invitrogen) in blocking buffer ( $3 \%$ goat serum in $1 \times$ PBS with $0.2 \%$ Tween 20 ). After rinsing, sections were incubated for $2 \mathrm{~h}$ at room temperature in blocking buffer containing $0.2 \%$ anti-rabbit antibody conjugated to peroxidase. Sections were rinsed again and incubated with the chromogen $\mathrm{DAB}$ for 5-30 min. Sections were then mounted on gelatin-coated slides, air-dried overnight, and coverslipped.

\section{Anatomical data acquisition and analysis}

Ipsilateral CS axon terminations. The Neurolucida tracing system (MBF Bioscience, Williston, VT) was used to measure axon length and varicosity number. These measurements were then used to create topographical density maps to determine changes in ipsilateral axon terminations. Axon length and varicosity number were measured in five transverse sections for each animal. For construction of topographical density maps ("heat maps"), digital tracings of individual sections were corrected for orientation and aligned with one another according to fiduciary marks (intersection between the gray matter above the central canal and the dorsal median septum) before being exported and quantified using custom programs written in the Matlab (MathWorks, Natick, MA) programming environment. Briefly, Neurolucida output files of individual sections of the spinal gray matter with traced axons were divided into $52 \times 52 \mu \mathrm{m}$ square regions of interest (ROIs). For each ROI, we computed the mean density of traced axons or marked varicosities. All axonal tracings corresponded to lines one pixel thick; varicosities were one pixel. This corresponds to the amount of label (i.e., total number of pixels) in the ROI divided by the area of the ROI. A matrix of mean axon or varicosities density was generated in Matlab that preserved the mediolateral and dorsoventral dimensions of the distribution of label in the gray matter. We quantified the regional axon and bouton density to generate maps of regional distribution of axonal label distribution and to quantify the amounts of label in different spinal laminas. These regions were determined by overlaying outlines of Rexed's laminas on the regional distribution maps and specifying matrix coordinates. Density is represented according to a color scale, from the lowest density (blue) to the highest (red). Regional distribution maps were generated for individual animals and then averaged for all animals within each treatment group.

Our anatomical data were corrected for the small variability in tracing differences between animals by dividing individual section data by the average number of ipsilateral axons per animal, counted in ipsilateral white matter tracts (ipsilateral dorsal column and ipsilateral ventral medial funiculus). This is the same correction factor used in other studies (Thallmair et al., 1998). Statistical analyses (Statview, for Macintosh) were performed on averaged data from individual animals in each group with ANOVA measure and Student's $t$ test with a Bonferroni/Dunn post hoc correction for the number of comparisons. We verified that the number of BDA-labeled ipsilateral axons within the dorsal and ventral white matter columns across did not differ between groups. Moreover, there were no significant differences in gray matter area (mean = $1,135,244.6 \pm 1840 \mu \mathrm{m}^{2}$ ) between groups (ANOVA, $F=0.758 ; p=$ $0.536)$.

The number of axons crossing the midline spinal gray matter was estimated using a method adapted from Zhou et al. (2003). Briefly, sections (7-12 per animal) were visualized using differential interference contract microscopy at $400 \times$. Dorsoventrally oriented lines were drawn at midline (determined by alignment with the midpoint of the dorsal columns, central canal, and ventral median fissure), and a parallel line 25 $\mu \mathrm{m}$ drawn lateral to midline on the ipsilateral hemifield using the Neurolucida program (see Fig. 5, inset). The number of axons within the gray matter between the two lines was counted. Averages for each animal were determined, and group averages were analyzed by ANOVA and post hoc comparisons with Bonferroni/Dunn corrections.

Contralateral CS axon terminations. To evaluate changes in axon distribution in the contralateral gray matter, a high-resolution photographic technique was used. Photomicrographs of each contralateral gray matter field ( $n=4$ sections per animal; 3 animals per group) were acquired through the microscope (at 200X) with the Neurolucida Virtual Slice module. Images were corrected for staining differences by adjusting the threshold control (Photoshop, Adobe, San Jose, CA) so that background staining was eliminated. Resulting black and white images were then exported to MATLAB, and the axon label density was analyzed using the same heat map algorithm and statistical analyses as those used for ipsilateral axon length measurements (above).

\section{Results}

To determine the contributions of activity- and injurydependent plasticity, we used a model of spared CST axons. Figure $1 A$ shows schematically the dense terminations of the predominantly contralateral projections of CS axons in each PT, and the sparse ipsilateral projections (Control). After unilateral PT lesion (Fig. $1 A$, Injured; red side eliminated) there is only sparse CS innervation on the side ipsilateral to the lesion. The dark pink territory marks the former termination field of lesioned contralateral CS axons. The light pink field on the contralateral side marks the former location of lesioned ipsilateral CS axons.

Four groups of animals were studied: untreated controls ( $n=$ $9)$; PT stimulation only $(n=7)$; PT lesion only $(n=8)$; and combined PT lesion and stimulation of the intact PT $(n=8)$. All lesions were made at the level of the medullary pyramid and were found to be complete on postmortem examination (supplemental Fig. S1, available at www.jneurosci.org as supplemental material). We assayed changes in CST connectivity by electrically stimulating the PT and recorded evoked motor responses from the deep radial nerve. We determined changes in the pattern and density of CST axon terminations by anterogradely tracing projections from motor cortex to the cervical enlargement.

\section{Chronic PT stimulation strengthens CS tract connections with spinal motor circuits}

We determined the effect of injury and/or chronic PT stimulation on the capacity of the CS tract to activate spinal motor circuits. 
A. Corticospinal tract
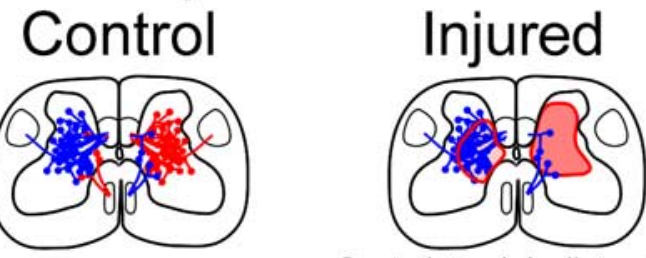

Contralateral Ipsilateral

B. Ipsilateral response

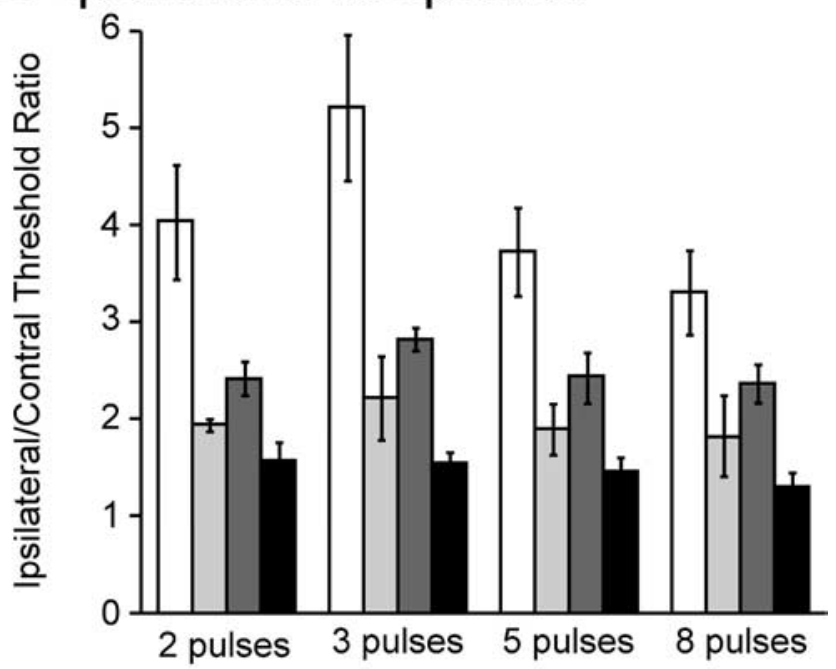

\section{Percent control}

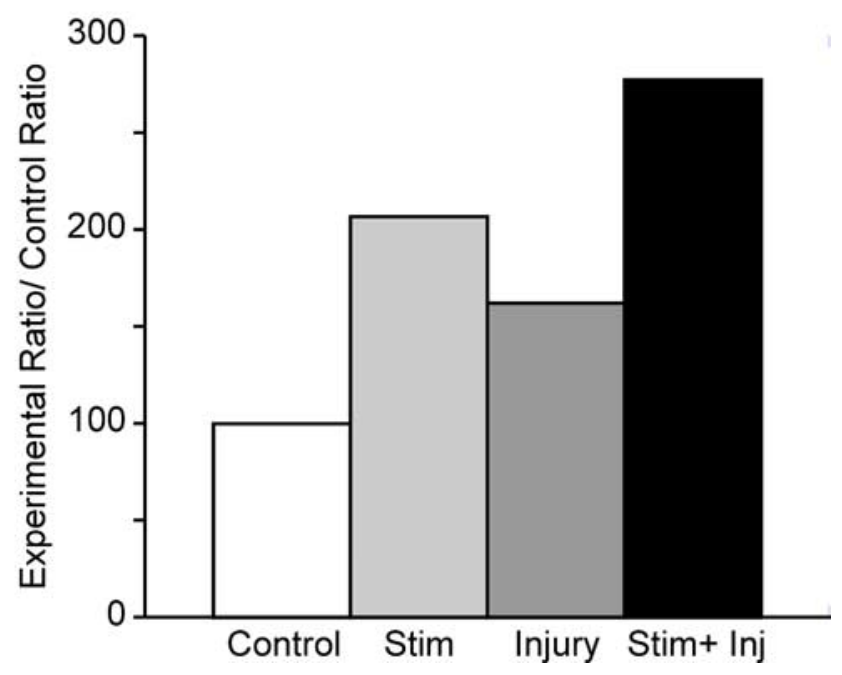

Figure 1. Chronic PT stimulation or injury alone increase the strength of ipsilateral connections, which were further strengthened by combined injury and stimulation. $\boldsymbol{A}$, Schematic model of CS termination field in the normal cervical spinal cord (Control) and the changes in termination density after PT lesion (Injured). Blue axons derive from the intact PT; red axons derive from the injured PT; pink shading in injured CS shows the former position of the lesioned axons. $\boldsymbol{B}$, Ratios of ipsilateral current threshold to contralateral current threshold (Ipsilateral/ Contral Threshold Ratio) needed to evoke responses in the deep branch of the radial nerve to PT stimulus trains of two, three, five, and eight pulses. A lower value in the ipsilateralcontralateral ratio indicates augmented access to ipsilateral spinal motor circuits. C, Summary graph showing percentage change in ipsilateral-contralateral threshold ratio. The values for each group were calculated from an average threshold response to all stimulus train lengths. Stimulation (Stim) and injury alone both show increased enhanced capacity to evoke ipsilateral motor responses, which is augmented further in the combined condition (Stim + Inj).
Control and injury alone animals received a PT electrode but were not stimulated. The stimulation and combined stimulation and injury groups had PT electrodes implanted and $10 \mathrm{~d}$ of daily stimulation. At the end of the stimulation or survival period, we conducted a terminal electrophysiology experiment. We used the implanted ventral PT electrode to evoke contralateral and ipsilateral forelimb motor responses, recorded using nerve cuff electrodes on the DRNs, and determined threshold currents. The threshold is defined as the minimum amount of current for a particular number of stimulus pulses to evoke an ENG response in the DRN. CST axons terminate extensively in the gray matter region containing DRN and other forelimb motoneurons (Liang et al., 1991). Short trains (two to eight pulses) of biphasic stimuli were used to evoke threshold responses on each side (supplemental Fig. S2, available at www.jneurosci.org as supplemental material). Contralateral responses were evoked at lower current thresholds than ipsilateral responses, as predicted by the density of CS terminations. We computed the ratio of ipsilateral/contralateral thresholds as a measure of the relative ability to activate ipsilateral spinal motor circuits.

Figure 1, $B$ and $C$, summarizes the results of the electrophysiology experiment. Control animals (Fig. $1 B$, open bars) $(n=5)$ have high ipsilateral/contralateral threshold ratios, indicating weak activation of ipsilateral spinal motor circuits (i.e., activation at higher stimulus strength). In contrast, each of the treated groups had better access to ipsilateral motor circuits. For each train of stimuli, the ipsilateral-to-contralateral threshold ratio was consistently much greater for the control animals than each of the treated groups (Fig. $1 B$ ) (ANOVA repeated measures, $F=$ 9.768; $p<0.003)$. There was a robust reduction in this ratio for stimulation alone (light gray bars; $n=3$ ) as well as for injury alone (dark gray bars; $n=3$ ). The largest reduction in the ipsilateral-to-contralateral threshold was seen in animals with combined injury and stimulation (black bars; $n=3$ ). Each animal group differed significantly from the other (Games-Howell post hoc test, $p<0.05$ ). To obtain a relative measure of the effects of activity and injury, and to compare the physiological and anatomical data, the multiple pulse data for each group were averaged and graphed as a percentage of the control ratio (Fig. 1C). Remarkably, relative to controls, there was approximately an additive effect of stimulation (107\%) and injury (62\%) separately in the combined stimulation and injured group (178\%). These findings show that injury-induced plasticity can be significantly augmented by chronic electrical stimulation of the CST.

\section{Ipsilateral CS axon terminal regional distribution}

We sought to determine whether the increased CS connectivity with ipsilateral motor circuits correlated with an increase in the density, or changes in the regional distribution, of CS axons in the ipsilateral spinal cord gray matter. We anterogradely labeled the CST by injecting BDA into the motor cortex. Ipsilateral CS tract axons are located within the ipsilateral dorsal and ventral columns (Joosten and Bar, 1999). The number of BDA-labeled ipsilateral axons within the dorsal and ventral white matter columns across all animal groups was $79.78 \pm 10.70$ (mean \pm SE). There were no significant differences in the number of descending ipsilateral axons between groups (ANOVA, $F=1.39 ; p=0.287$ ).

To determine changes in the topographic distribution of the ipsilateral CS projection, we traced all labeled ipsilateral axons on five randomly chosen transverse sections through the cervical enlargement. Averaged regional distributions of ipsilateral CS axons are shown in Figure $2 \mathrm{~A}$. Label density within defined regions of interest is coded according to a color scale (blue, least 


\section{A. Axon}

A1

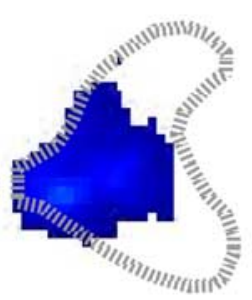

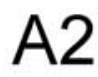

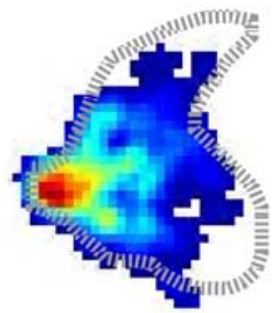

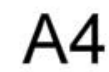
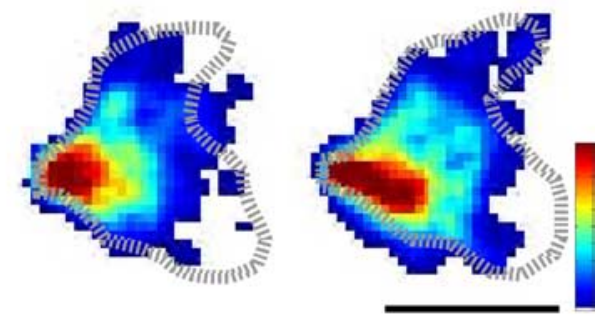

B. Varicosities B1
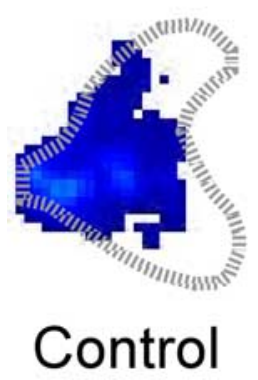
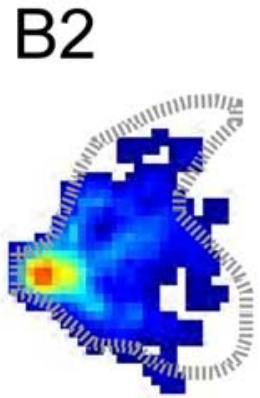

Stim
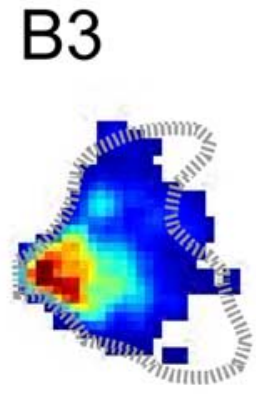

Injury
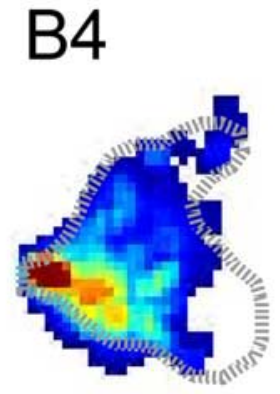

Inj+Stim

Figure 2. Stimulation and injury each promote increased density of ipsilateral CS terminations, which is further augmented in the combined condition. $\boldsymbol{A}$, Axon density (terminals and preterminal axons) maps. $\boldsymbol{B}$, Bouton density maps. Color coded average density maps are shown for controls $(\boldsymbol{A} \mathbf{B}, \boldsymbol{B} \mathbf{n}, n=4)$, animals with PT stimulation alone (Stim; $\boldsymbol{A 2}, \boldsymbol{B 2} ; n=4)$, animals with injury (PTx) alone $(A 3, B 3 ; n=5)$, and animals with both stimulation and injury $(\operatorname{Inj}+\operatorname{Stim} ; \boldsymbol{A} 4, B \mathbf{B} ; n=5)$. The ipsilateral gray matter border (gray hatched line) shows averaged borders from the individual animals in the group. Calibration: $500 \mu \mathrm{m}$; Color scale: $A$, $0-5.7 \mu \mathrm{m}$ axon $/ \mu \mathrm{m}^{2}$ area; $\boldsymbol{B}, 0-0.7$ boutons $/ \mu \mathrm{m}^{2}$ area.

dense; red, most dense). As others have reported (Brosamle and Schwab, 1997; Lacroix et al., 2004), in our control animals ipsilateral CS axons terminated most densely within the medial portion of the intermediate zone of the gray matter (Fig. 2A1) (average of four rats). We determined the effects of stimulation and injury first separately and then combined. Stimulation on average increased the local density of terminations as well as the overall distribution within the gray mater (Fig. $2 A 2)(n=4)$. This shows that stimulation in maturity, as in development (Salimi et al., 2004), leads to greater outgrowth in the spinal gray matter. Injury alone (Fig. 2A3) $(n=5)$ resulted in a qualitatively similar augmentation, as for stimulation alone. Combined stimulation and injury (Fig. 2A4) $(n=5)$ resulted in greater augmentation of CS axon terminations than either stimulation or injury alone. Changes in the local density and topographic distribution of varicose portions of labeled CS axons, which are putative presynaptic sites (i.e., boutons), paralleled changes in CS axon terminations (Fig. $2 B$ ).

We quantified changes in the growth of ipsilateral CS tract axon terminations by summing the total length of labeled CS axons within the ipsilateral gray matter (Fig. $3 A$ ). Stimulation alone (light gray bar), compared with controls (white bar), resulted in a significant $80.6 \%$ increase in total axon length ( $t$ test; corrected for multiple comparisons: $p=0.011$ ). Injury alone (dark gray bar) resulted in a significant $117.1 \%$ increase in the total corrected axon length than controls $(p=0.003)$. Although higher than stimulation alone, this increase is not significant $(p=0.824)$. Combining $10 \mathrm{~d}$ of PT stimulation after injury resulted in the largest increase, relative to control, of $193.6 \%$ $(p=0.002)$. Similar to evoked ipsilateral motor responses, there was approximately an additive anatomical effect of stimulation and injury. Consistent with an additive effect, a two-factor ANOVA revealed that there were no significant interactions (i.e., neither synergy or decrement) between stimulation and injury ( $p=0.214)$. Injury and stimulation, alone and in combination, also produced increases in CS axon varicosity density, with an identical rank order of increases (one-ANOVA, $F=$ 3.480; $p<0.04)$.

To compare changes in the distribution between animal groups more directly, we transformed the topographic data into a one-dimensional plot of total ipsilateral CS labeling along the dorsoventral axis. Figure 4 plots the average axon density \pm SE for each group. Combined injury and stimulation (red lines) resulted in a greater amount of outgrowth into the ventral horn (arrow) than stimulation or injury alone. To examine the question of preferential ventral outgrowth further, we compared ventral horn termination density across groups (Fig. 3B). There was an overall significant group difference (ANOVA, $F=7.37 ; p=003$ ). Importantly, combined injury and stimulation was significantly greater than injury alone (unpaired $t$ test; $p=0.04$ ). As with ventral horn axons, there also was an overall significant group difference in axon varicosities in the ventral horn (ANOVA, $F=4.53 p=0.02$ ), and there was a significant increase in the combined stimulation and injury group compared with controls (unpaired $t$ test; $p=0.03$ ). Our findings show that stimulation augments injury-dependent changes, with preferential outgrowth into the ventral horn.

\section{Lamina 10 decussating axons}

Contralateral CS axons cross the midline (i.e., recross) at the segmental level to make an important contribution to CS axons in the ipsilateral gray matter (Brosamle and Schwab, 1997). To estimate changes in the number of axons that cross the midline at the segmental level, we counted the number of ipsilateral axons in lamina 10, $25 \mu \mathrm{m}$ from the midline (Fig. 5, inset). In control animals, there is a small number of CS axons in this region (mean $=1.51 \pm 0.17$ axons $/ 40 \mu \mathrm{m}$ section) (Fig. 5, open bar). Stimulation alone increased the number of axon (mean $=4.4 \pm$ 1.2 axons $/ 40 \mu \mathrm{m}$ section) (Fig. 5, light gray bar), as did injury alone $($ mean $=4.13 \pm 1.5$ axons $/ 40 \mu \mathrm{m}$ section) (Fig. 5 , dark gray bar). Combined, injury plus stimulation resulted in the most CS axons in this area (mean $=7.10 \pm 1.01$ axons $/ 40 \mu \mathrm{m}$ section) (Fig. 5, black bar). We found that there was a significant overall trend across the four groups of animals (one-way ANOVA, $F=$ 6.833; $p=0.005$ ). Post hoc testing revealed that the difference between control and combined injury and stimulation groups was significantly different (Bonferroni-Dunn; $p=0.0005$ ). These finding suggest that recrossing segmental CS axons, as for ipsilat- 


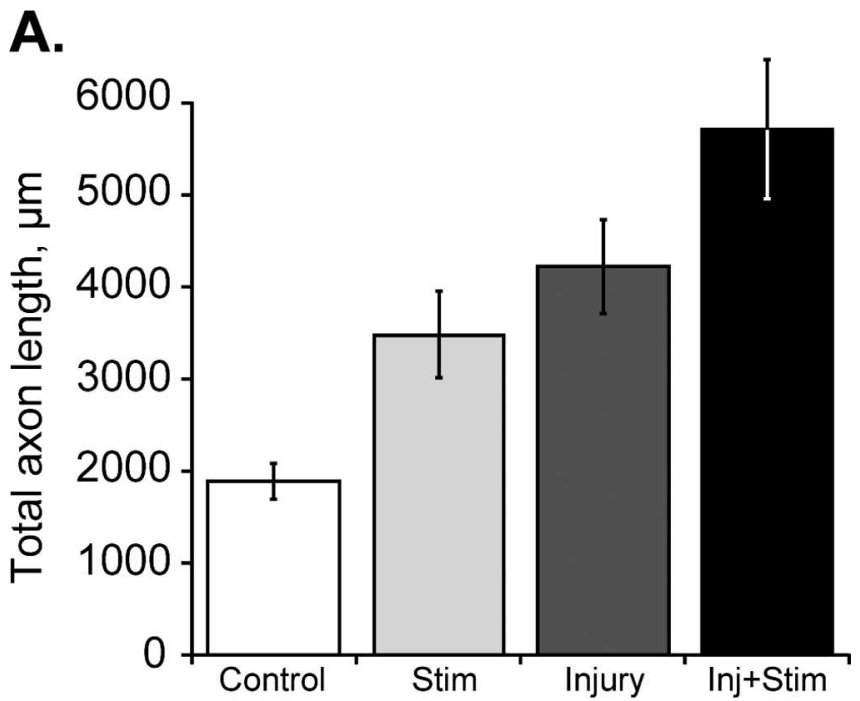

B.

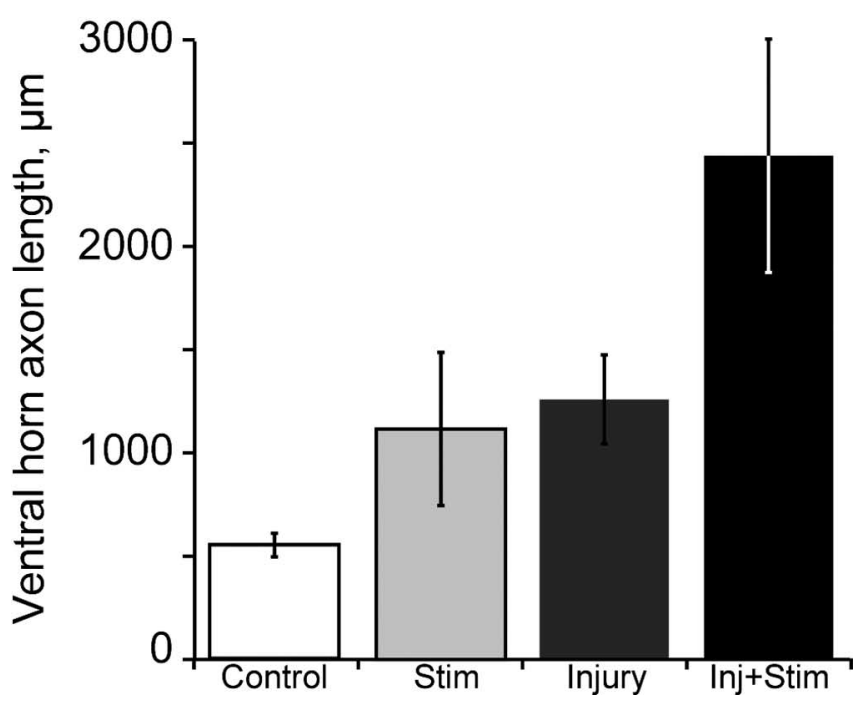

Figure 3. Stimulation (Stim) and injury alone and in combination (Inj+Stim) augment total ipsilateral CS termination axon length. $\boldsymbol{A}$, Total average ipsilateral axon length in the controls, stimulation alone, injury alone, and combined injury and stimulation. Stimulation ( $p=0.011$ ) and injury ( $p=0.003$ ) alone each augmented total axon length significantly compared with control. Combined, there was a larger increase $(p=0.002)$. $p$ values were calculated from $t$ test with Bonferroni/Dunn correction. $\boldsymbol{B}$, Ventral ipsilateral axon length in the controls, stimulation alone, injury alone, and combined condition (ANOVA, $F=7.37 ; p=003$ ). Combined stimulation and injury significantly augmented axon length in this region compared with injury alone $(t$ test; $p=0.04)$.

eral CS axons generally, showed the greatest increase for the combined injury plus stimulation group. Although the absolute values are small, it should be noted that these numbers are for $40 \mu \mathrm{m}$ thin sections. The cervical enlargement (C5-C8) is $\sim 7 \mathrm{~mm}$ (Alstermark et al., 2004). Extrapolating the range of axons we observed on $40 \mu \mathrm{m}$ sections, we would expect $\sim 1242$ axons in the combined injury and stimulation group.

\section{Contralateral CS axon distribution}

Unilateral PT lesion and PT stimulation will have bilateral effects in the spinal cord, mediated by the sparse ipsilateral terminations and the dense contralateral terminations (Fig. $1 A$ ). We showed that the ipsilateral terminations react strongly to injury and stimulation alone and in combination. We predicted that contralat-

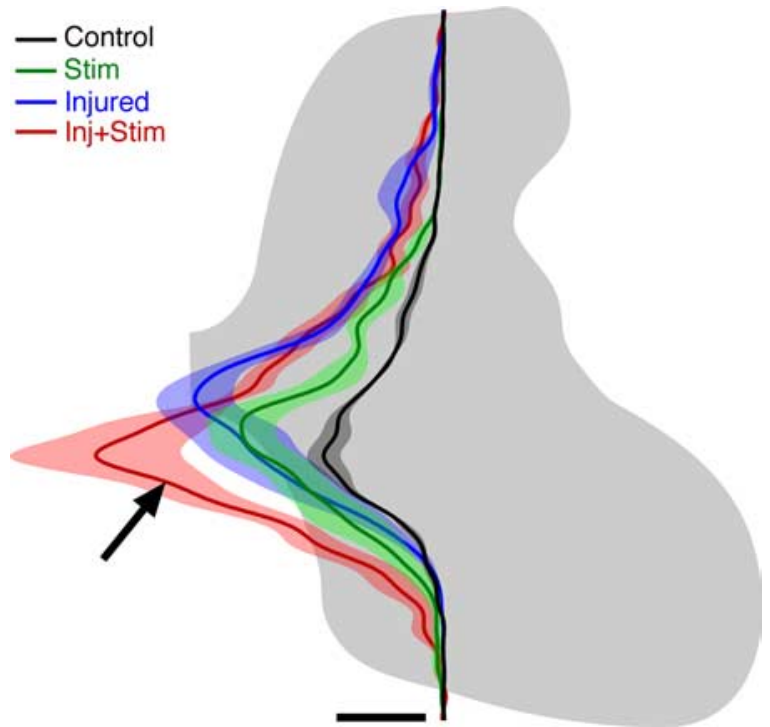

Figure 4. Stimulation after injury shifts the distribution of CS terminations ventrally in the gray matter. Dorsoventral distributions of mean axon density from the dorsal to the ventral borders of the ipsilateral gray mater are shown. Each line plots the average density of axon terminations (preterminal and terminal axons; no boutons) of the animals in each group; light shading plots \pm SEM. Data from controls are shown in black ( $n=4$ rats); stimulation alone (Stim; $n=4)$, green; injury alone $(n=5)$, blue; and combined injury and stimulation $(\operatorname{Inj}+\operatorname{Stim} ; n=5)$, red. The arrow indicates a ventral location where axon length is greatest in the injury and stimulation group. To normalize for differences in the size of the gray matter across animals, all graphs were interpolated to 1000 points between the dorsal and ventral margins of the gray matter. Calibration: $30 \mu \mathrm{m}$ of labeled axons.

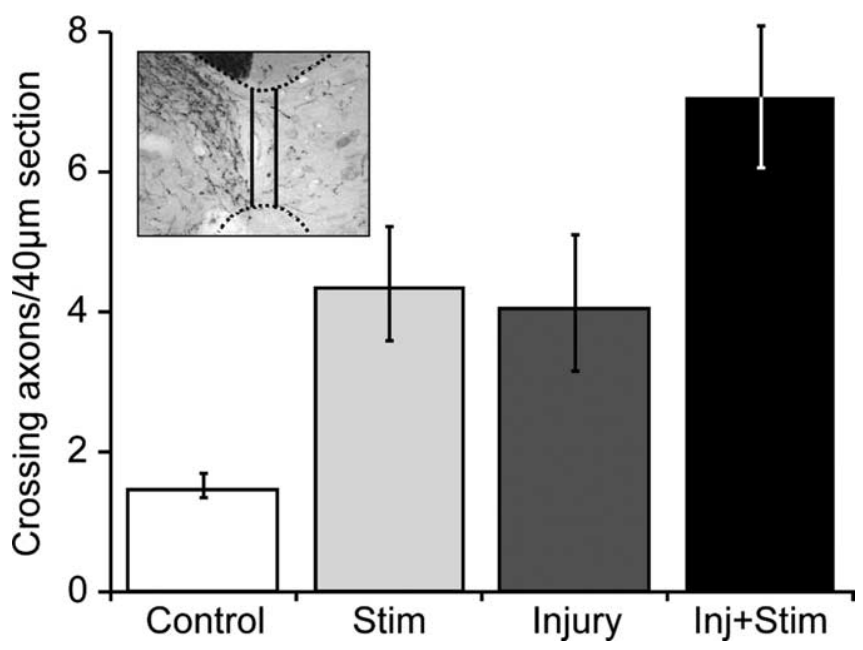

Figure 5. Stimulation and injury alone and combination augment the number of axons crossing in the cervical spinal gray matter. Crossing axon counts per 40- $\mu$ m-thick transverse section for control, stimulation (Stim), injury, and combined stimulation and injury (Inj+Stim). There was a significant difference in lamina 10 axons between groups (ANOVA, $F=6.83 ; p=$ 0.005 ), and importantly, combined stimulation and injury significantly augmented crossing axons compared with injury alone (Bonferroni/Dunn post hoc, $p=0.0005$ ). Inset, Representative example of axons in lamina 10 of the gray matter. Solid vertical lines indicate the midline (left) and $25 \mu \mathrm{m}$ ipsilateral to midline (right), and dashed lines mark the dorsal and ventral gray matter borders. Only axons within the $25 \mu \mathrm{m}$ region were counted.

eral projections, because of their high density, would create greater competition among themselves and therefore have a less robust outgrowth than the sparse ipsilateral terminations. It was not possible to trace the contralateral CS axons individually, as we did for the ipsilateral terminations, because they were too dense. Instead, we developed a measure of axon labeling based on opti- 


\section{A. Contralateral axon distribution}

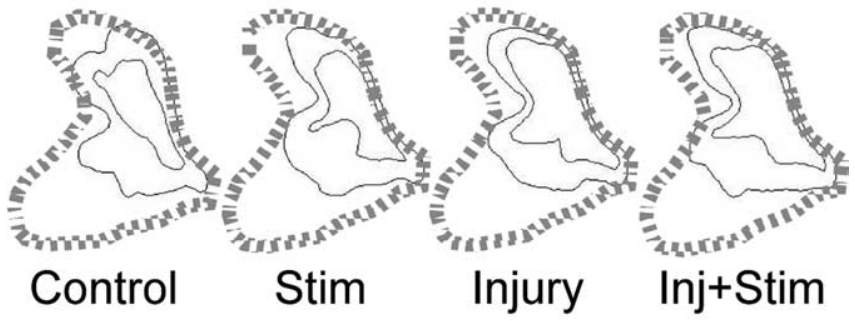

\section{B. Contralateral gray matter label}

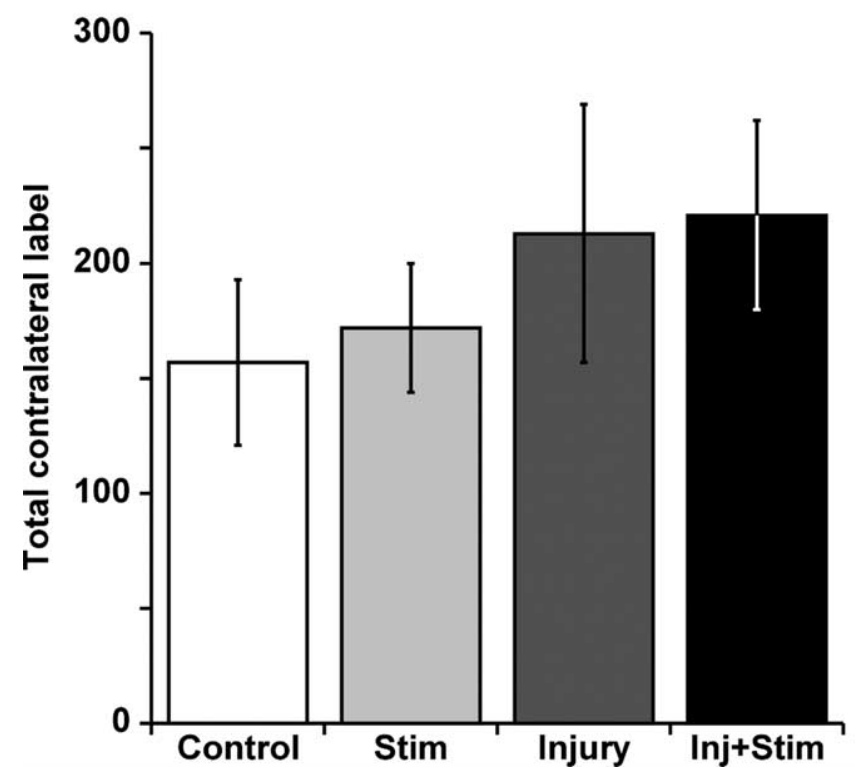

Figure 6. Contralateral CS terminations do not respond significantly to stimulation (Stim) and injury alone and in combination (Inj+Stim). $\boldsymbol{A}$, Distributions of regions of densest CS labeling (inner solid line; 35\%) and total labeling (outer solid line; total label). Axon label was determined by optical density after reducing background signal to zero. Data from the four animal groups are shown: controls $(n=4)$, stimulated alone $(n=4)$, injury alone $(n=5)$, and combined injury and stimulation $(n=5)$. $\boldsymbol{B}$, Total contralateral label for each animal group. There were no significant differences between groups (ANOVA, $F=0.56 ; p=0.655$ ).

cal density that took advantage of the high contrast between labeled CS axons unstained gray matter background (see Materials and Methods). Using this approach, we found the region of densest labeling (35\% of maximal) to have expanded slighted (Fig. $6 \mathrm{~A}$, solid inner line), as did the total labeled region (solid outer line). The total amount of label (Fig. 6B) showed a modest and not significantly increased trend toward increased labeling in the stimulation only, lesion only, and combined groups (one-way ANOVA, $F=0.562 ; p=0.655)$. Thus, although there was a trend toward similar outgrowth effects on the contralateral axon terminations, the magnitude was much smaller than for the ipsilateral terminations and was not significantly different.

\section{Discussion}

There is an important, and largely unrecognized, interplay between activity and injury in the mature CS system. Promoting CS neural activity strongly promoted ipsilateral connections, but without changing the overall pattern of growth in the spinal gray matter. Injury alone had a similar effect. Activity and injury, in combination, complemented each other to produce the strongest connections. Furthermore, the combination led to a pattern of ipsilateral CS terminations that was preferentially directed to the ventral spinal cord, a new pattern of connections that is apt to be adaptive in supporting motor behaviors mediated by the motor cortex. In animals with combined injury and stimulation, ipsilateral motor responses evoked by $\mathrm{PT}$ stimulation rivaled those on the contralateral side, yet the density of ipsilateral terminations was much lower than contralateral terminations. This suggests that most of the increased ipsilateral response is mediated by enhancing synaptic strength. Although strengthening ipsilateral connections is the most parsimonious explanation, it is plausible that contralateral connections to spinal commissural neurons could serve this purpose. Either way, it shows that the motor cortex can likely access ipsilateral spinal motor circuits.

\section{Reactive sprouting of spared axons after injury}

We found a significant increase in the length of spared ipsilateral CST axons after PT lesion. This increase is likely caused by a combination of local sprouting of preexisting ipsilateral terminations and increased numbers of contralateral CS axons that recross the midline within lamina 10. Compared with previous studies that quantified reactive sprouting of intact CS axons after partial injury, we report substantially more growth. This is likely attributable to our approach of monitoring all labeled ipsilateral CS axons, which may be a more sensitive assay than more limited counting strategies (Thallmair et al., 1998; Weidner et al., 2001). One study that measured reactive sprouting in the monkey after spinal hemisection used retrograde spinal labeling to show a $24 \%$ increase in the numbers of labeled ipsilateral CST neurons in the cortex (Aoki et al., 1986), which is consistent with our findings.

The pattern of spared axon growth after unilateral pyramidotomy fits with our competition model. We found that after removal of the major source of CS input, the contralateral CS tract, the motor effects of ipsilateral stimulation were significantly strengthened, and CS outgrowth within the ipsilateral gray matter was robust. In contrast, removal of a minor source of CS input, the ipsilateral CS tract, did not result in either stronger contralateral motor effects or significant axon outgrowth. Although our anatomical assay for monitoring contralateral terminations is less sensitive than for ipsilateral terminations, the physiological assay is not. Promoting ipsilateral, not contralateral, CS connectivity suggests that the efficacy of sprouting depends on competition among spared axons. Efficacy appears to be greater when a large component of competing CS terminations are removed.

A question that arises from this model is whether other descending or spinal afferent systems sprout in reaction to CS injury. Interestingly, sprouting by rubrospinal axons, in otherwise untreated animals, was not reported after pyramidotomy (Raineteau et al., 2002). Rather, such sprouting was only reported for animals treated with the IN-1 anti-NOGO antibody. Although this needs to be examined directly, these findings suggest that interactions between like axons are stronger than between axons of different neural systems. Understanding the change in pattern of terminations from different neural systems after injury will help us to formulate strategies to restore functional circuits, perhaps by selectively stimulating certain systems and not others.

\section{Activity-dependent competition between mature CS axon terminations}

Our present findings in the adult animal extend our developmental findings of the importance of activity-dependent competition (Martin, 2005). In the kitten, we found that active CS axons over- 
grow termination fields of inactive or less active axons. A hint about activity's role in maturity comes from our recent work in the cat showing that activity-dependent competition can be manipulated late in development, after the pattern of CS connections is stable, to reverse aberrant CS connectivity (Friel and Martin, 2007). It has been suggested that activity-dependent competition, as we have shown in the cat, drives development of CS projections in the human (Eyre et al., 2007).

Why were activity-dependent processes more effective in promoting ipsilateral than contralateral CS terminations? Although activity could promote sprouting of spared axons in maturity in a variety of ways [for review, see Qiu et al. (2002) and David and Lacroix (2003)], stable axon outgrowth likely requires synapse formation (Nelson et al., 1995; Turrigiano and Nelson, 2000) with spinal target neurons. And the capacity for new synapses depends critically on activity-dependent competition (Hua et al., 2005). Akin to what we described above for injury-induced sprouting, in the intact animal, ipsilateral terminations are sparse, which assures a low probability of competition. Thus, the potential for forming new ipsilateral connections may be high. In contrast, contralateral termination density is normally high, making it a more competitive environment and limiting its capacity to form new or stronger connections.

\section{Interplay between injury- and activity-dependent plasticity}

Remarkably, injury and stimulation promote outgrowth and strength of ipsilateral motor responses in a quantitatively similar way. Combined, injury- and activity-dependent plasticity were additive. Not surprisingly, there also was a parallel increase in the density and extent of axon varicosities, which are presumably presynaptic boutons. It is plausible that whatever drives axon outgrowth and synapse formation under these two different conditions has separate mechanisms that simply add. Alternatively, our finding of strong activity-dependent outgrowth and strengthening in the intact CS system raises a second possibility that a component of the response to injury is a response to decreased activity in the damaged system. The injured CS system becomes a less used (Jones and Schallert, 1994), and presumably less active, component of the motor systems. This could disinhibit the contralateral motor cortex, because there are strong reciprocal inhibitory interactions between the motor cortices in each hemisphere (Ferbert et al., 1992), and lead to an increase in the activity of spared axons. Thus, unilateral lesion of the CS system could lead to an increase in activation of the contralateral, intact, CS system by transcallosal disinhibition.

Combining stimulation and injury led to a novel pattern of terminations in the ventral horn, and thus the opportunity to form novel ventral horn connections. This must be an activitydependent effect that is specific to this model of spared axons, because neither injury alone nor stimulation alone biased terminal outgrowth ventrally. Strengthening ventral connections could improve motor control by two mechanisms. First, the new terminations could more directly access spinal motor neurons, either by direct contact (Weidner et al., 2001) or through interneurons. Second, these ventral terminations may abrogate the spasticity that is seen after CS injury. Spasticity involves a sensitized stretch reflex that could be better regulated by increased CS innervation of ventral motor circuits (Pierrot-Deseilligny and Burke, 2005).

\section{Harnessing activity- and injury-dependent processes to reshape CS motor circuitry after injury}

CS tract stimulation is a novel, and a remarkably circuit-specific, way to promote motor pathway connectivity after brain or spinal injury. Previous efforts have focused largely on neutralizing or abrogating growth inhibitory factors, either at the site of injury or in termination fields (Bradbury et al., 2002; Qiu et al., 2002; McGee and Strittmatter, 2003; Schwab, 2004; Massey et al., 2006), which augments the connectivity of all local and responsive neural systems. This strategy can promote functional improvement (Thallmair et al., 1998; Merkler et al., 2001; GrandPre et al., 2002), but it also may result in aberrant connectivity that could produce pain (Hofstetter et al., 2005) or spasticity. Locomotorbased behavioral strategies (Edgerton et al., 2004) and functional electrical stimulation (FES) (Gorman, 2000) produce a generalized increase in activity in the segments stimulated. Our approach of selectively stimulating the CS system, in contrast, would be expected to activate preferentially spinal motor circuits that mediate cortical control. Thus, it is likely that generalized "bottom-up" approaches such as locomotor training and FES will strengthen a different (Huang et al., 2000), but overlapping, set of spinal circuits than CS system stimulation (Curfs et al., 1996). Importantly, the "top-down" approach of CS system stimulation allows activity-dependent processes to be focused on the specific cortical and spinal circuits that are most important for voluntary motor control (Pierrot-Deseilligny and Burke, 2005).

Just as injury and activity produced complementary effects on CS connectivity in this study, combining CS stimulation with other efforts to promote axon outgrowth will also likely complement one another. Severed CS axons that grow through a lesion site after the application of factors that reduce axon growth inhibition (McGee and Strittmatter, 2003; Schwab, 2004) might be directed to form more adaptive and stronger circuits if they are also electrically stimulated. Likewise, reducing axon growth inhibition locally in the spinal cord might improve the outgrowth that we saw in spared axon terminations. Thus, in addition to better understanding the relationship of injury and activity in the mature CS system, we have introduced a new strategy that could add significantly to the existing efforts to restore function after CNS injury.

\section{References}

Alstermark B, Ogawa J, Isa T (2004) Lack of monosynaptic corticomotoneuronal EPSPs in rats: disynaptic EPSPs mediated via reticulospinal neurons and polysynaptic EPSPs via segmental interneurons. J Neurophysiol 91:1832-1839.

Aoki M, Fujito Y, Satomi H, Kurosawa Y, Kasaba T (1986) The possible role of collateral sprouting in the functional restitution of corticospinal connections after spinal hemisection. Neurosci Res 3:617-627.

Bareyre FM, Kerschensteiner M, Raineteau O, Mettenleiter TC, Weinmann O, Schwab ME (2004) The injured spinal cord spontaneously forms a new intraspinal circuit in adult rats. Nat Neurosci 7:269-277.

Bradbury EJ, Moon LD, Popat RJ, King VR, Bennett GS, Patel PN, Fawcett JW, McMahon SB (2002) Chondroitinase ABC promotes functional recovery after spinal cord injury. Nature 416:636-640.

Brosamle C, Schwab ME (1997) Cells of origin, course, and termination patterns of the ventral, uncrossed component of the mature rat corticospinal tract. J Comp Neurol 386:293-303.

Brosamle C, Schwab ME (2000) Ipsilateral, ventral corticospinal tract of the adult rat: ultrastructure, myelination and synaptic connections. J Neurocytol 29:499-507.

Curfs MH, Gribnau AA, Dederen PJ, Bergervoet-Vernooij IW (1996) Induction of c-fos expression in cervical spinal interneurons after kainate stimulation of the motor cortex in the rat. Brain Res 725:88-94.

David S, Lacroix S (2003) Molecular approaches to spinal cord repair. Annu Rev Neurosci 26:411-440. 
Edgerton VR, Tillakaratne NJ, Bigbee AJ, de Leon RD, Roy RR (2004) Plasticity of the spinal neural circuitry after injury. Annu Rev Neurosci 27:145-167.

Eyre JA, Smith M, Dabydeen L, Clowry GJ, Patacchi E, Battini R, Guzzetta A, Cioni G (2007) Is hemiplegic cerebral palsy equivalent to amblyopia of the corticospinal system? Ann Neurol 62:493-503.

Ferbert A, Priori A, Rothwell JC, Day BL, Colebatch JG, Marsden CD (1992) Interhemispheric inhibition of the human motor cortex. J Physiol (Lond) 453:525-546.

Friel K, Martin JH (2005) Role of sensory-motor cortex activity in postnatal development of corticospinal axon terminals in the cat. J Comp Neurol 485:43-56.

Friel K, Martin JH (2007) Bilateral activity-dependent interactions in the developing corticospinal system. J Neurosci 10:11083-11090.

Friel KM, Drew T, Martin JH (2007) Differential activity-dependent development of corticospinal control of movement and final limb position during visually-guided locomotion. J Neurophysiol 97:3396-3406.

Gorman PH (2000) An update on functional electrical stimulation after spinal cord injury. Neurorehabil Neural Repair 14:251-263.

GrandPre T, Li S, Strittmatter SM (2002) Nogo-66 receptor antagonist peptide promotes axonal regeneration. Nature 417:547-551.

Hicks RG, Woodforth IJ, Crawford MR, Stephen JP, Burke DJ (1992) Some effects of isoflurane on I waves of the motor evoked potential. Br J Anaesth 69:130-136.

Hill CE, Beattie MS, Bresnahan JC (2001) Degeneration and sprouting of identified descending supraspinal axons after contusive spinal cord injury in the rat. Exp Neurol 171:153-169.

Hofstetter CP, Holmstrom NA, Lilja JA, Schweinhardt P, Hao J, Spenger C, Wiesenfeld-Hallin Z, Kurpad SN, Frisen J, Olson L (2005) Allodynia limits the usefulness of intraspinal neural stem cell grafts; directed differentiation improves outcome. Nat Neurosci 8:346-353.

Hua JY, Smear MC, Baier H, Smith SJ (2005) Regulation of axon growth in vivo by activity-based competition. Nature 434:1022-1026.

Huang A, Noga BR, Carr PA, Fedirchuk B, Jordan LM (2000) Spinal cholinergic neurons activated during locomotion: localization and electrophysiological characterization. J Neurophysiol 83:3537-3547.

Jones TA, Schallert T (1994) Use-dependent growth of pyramidal neurons after neocortical damage. J Neurosci 14:2140-2152.

Joosten EA (1997) Corticospinal tract regrowth. Prog Neurobiol 53:1-25.

Joosten EA, Bar DP (1999) Axon guidance of outgrowing corticospinal fibres in the rat. J Anat 194(Pt 1):15-32.

Julien C, Rossignol S (1982) Electroneurographic recordings with polymer cuff electrodes in paralyzed cats. J Neurosci Methods 5:267-272.

Lacroix S, Havton LA, McKay H, Yang H, Brant A, Roberts J, Tuszynski MH (2004) Bilateral corticospinal projections arise from each motor cortex in the macaque monkey: a quantitative study. J Comp Neurol 473:147-161.

Liang F, Moret V, Wiesendanger M, Rouiller EM (1991) Corticomotoneuronal connections in the rat: evidence from double-labeling of motoneurons and corticospinal axon arborizations. J Comp Neurol 311:356-366.
Martin JH (2005) The corticospinal system: from development to motor control. Neuroscientist 11:161-173.

Martin JH, Lee S (1999) Activity-dependent competition between developing corticospinal terminations. NeuroReport 10:2277-2282.

Massey JM, Hubscher CH, Wagoner MR, Decker JA, Amps J, Silver J, Onifer SM (2006) Chondroitinase ABC digestion of the perineuronal net promotes functional collateral sprouting in the cuneate nucleus after cervical spinal cord injury. J Neurosci 26:4406-4414.

McGee AW, Strittmatter SM (2003) The Nogo-66 receptor: focusing myelin inhibition of axon regeneration. Trends Neurosci 26:193-198.

Meng Z, Li Q, Martin JH (2004) The transition from development to motor control function in the corticospinal system. J Neurosci 24:605-614.

Merkler D, Metz GA, Raineteau O, Dietz V, Schwab ME, Fouad K (2001) Locomotor recovery in spinal cord-injured rats treated with an antibody neutralizing the myelin-associated neurite growth inhibitor Nogo-A. J Neurosci 21:3665-3673.

Neafsey EJ, Bold EL, Haas G, Hurley-Gius KM, Quirk G, Sievert CF, Terreberry RR (1986) The organization of the rat motor cortex: a microstimulation mapping study. Brain Res 396:77-96.

Nelson PG, Fields RD, Liu Y (1995) Neural activity, neuron-glia relationships, and synapse development. Perspect Dev Neurobiol 2:399-407.

Paxinos G, Watson C (1986) The rat brain in stereotaxic coordinates, Ed 2. Sydney: Academic.

Pierrot-Deseilligny E, Burke D (2005) The circuitry of the human spinal cord. Cambridge, UK: Cambridge UP.

Qiu J, Cai D, Filbin MT (2002) A role for cAMP in regeneration during development and after injury. Prog Brain Res 137:381-387.

Raineteau O, Fouad K, Bareyre FM, Schwab ME (2002) Reorganization of descending motor tracts in the rat spinal cord. Eur J Neurosci 16:1761-1771.

Salimi I, Friel K, Martin JH (2004) Plasticity of corticospinal axon terminals produced by pyramidal tract stimulation or sensorimotor cortex inactivation in mature cats. Soc Neurosci Abstr 30:878.7.

Schwab ME (2004) Nogo and axon regeneration. Curr Opin Neurobiol 14:118-124.

Sloan TB (1998) Anesthetic effects on electrophysiologic recordings. J Clin Neurophysiol 15:217-226.

Thallmair M, Metz GAS, Z’Graggen W, Raineteau O, Kartje GL, Schwab ME (1998) Neurite growth inhibitors restrict plasticity and functional recovery following corticospinal tract lesions. Nat Neurosci 1:124-131.

Turrigiano GG, Nelson SB (2000) Hebb and homeostasis in neuronal plasticity. Curr Opin Neurobiol 10:358-364.

Weidner N, Ner A, Salimi N, Tuszynski MH (2001) Spontaneous corticospinal axonal plasticity and functional recovery after adult central nervous system injury. Proc Natl Acad Sci USA 98:3513-3518.

Zhou L, Baumgartner BJ, Hill-Felberg SJ, McGowen LR, Shine HD (2003) Neurotrophin-3 expressed in situ induces axonal plasticity in the adult injured spinal cord. J Neurosci 23:1424-1431. 